\title{
EXISTENCE OF SOLUTIONS TO EVOLUTION SECOND ORDER HEMIVARIATIONAL INEQUALITIES WITH MULTIVALUED DAMPING
}

\author{
Zdzisław Denkowski * \\ Jagiellonian University \\ Faculty of Mathematics and Computer Science \\ Institute of Computer Science \\ ul. Nawojki 11, PL-30072 Krakow, Poland \\ denkowski@softlab.ii.uj.edu.pl \\ Stanisław Migórski * \\ Jagiellonian University \\ Faculty of Mathematics and Computer Science \\ Institute of Computer Science \\ ul. Nawojki 11, PL-30072 Krakow, Poland \\ migorski@softlab.ii.uj.edu.pl
}

\begin{abstract}
In this paper we examine an evolution problem which describes the dynamic contact of a viscoelastic body and a foundation. The contact is modeled by a general normal compliance condition and a friction law which are nonmonotone, possibly multivalued and of the subdifferential form while the damping operator is assumed to be coercive and pseudomonotone. We derive a formulation of the model in the form of a multidimensional hemivariational inequality. Then we establish the a priori estimates and the existence of weak solutions by using a surjectivity result.
\end{abstract}

Keywords: Contact problem, hemivariational inequality, subdifferential, damping, nonconvex, friction, hyperbolic, viscoelasticity.

*Supported in part by the State Committee for Scientific Research of the Republic of Poland (KBN) under Research Grants no. 2 P03A 00325 and 4 T07A 02726. 


\section{Introduction}

In this paper we investigate the class of evolution second order hemivariational inequalities. By a hemivariational inequality we mean an evolution variational inequality involving a nonmonotone multivalued map of the Clarke subdifferential type. The problem under consideration is as follows

$$
\left\{\begin{array}{l}
u^{\prime \prime}(t)+A\left(t, u^{\prime}(t)\right)+B u(t)+\partial J(t, u(t)) \ni f(t) \text { a.e. } t \in(0, T) \\
u(0)=u_{0}, u^{\prime}(0)=u_{1},
\end{array}\right.
$$

where $A:(0, T) \times V \rightarrow 2^{V^{*}}$ is a nonlinear multivalued damping operator, $V$ being a reflexive Banach space with its dual $V^{*}, B: V \rightarrow V^{*}$ is a bounded linear operator, not necessary coercive, $\partial J$ denotes the Clarke subdifferential of a locally Lipschitz function $J$ and $f, u_{0}$ and $u_{1}$ are prescribed data. The motivation for the study of the problem (1) comes from mechanics and engineering where hemivariational inequalities express the principle of virtual work or power, e.g. unilateral contact problems in nonlinear elasticity and viscoelasticity, problems describing frictional and adhesive effects, problem of delamination of plates, loading and unloading problems in engineering structures (cf. Panagiotopoulos [26-27] and Naniewicz and Panagiotopoulos [24]).

The notion of hemivariational inequality was introduced by P.D. Panagiotopoulos in the early eighties as variational expressions for several classes of mechanical problems with nonsmooth and nonconvex energy superpotentials. In the case of convex superpotentials the hemivariational inequalities reduce to variational inequalities considered earlier by many authors (see e.g. Duvaut and Lions [7] and the references therein). The recent mathematical results on the stationary hemivariational inequalities can be found in Naniewicz and Panagiotopoulos [24], Motreanu and Panagiotopoulos [23] and Haslinger et al. [12]. We refer to Migorski [20-22] and the references therein for the results on the first order evolution and parabolic hemivariational inequalities. We mention that the hemivariational inequalities of hyperbolic type were firstly considered by Panagiotopoulos $[29,28]$ who studied models involving the one dimensional reaction-velocity laws. The hyperbolic hemivariational inequalities with a multivalued relation depending on the first order derivative of the unknown function were treated by Goeleven et al. [10], Haslinger et al. [12], Gasiński [8], Migorski [19], while the hemivariational inequalities with a subdifferential term which depends on the unknown function were studied by Panagiotopoulos and Pop [30], Haslinger et al. [12], Gasiński and Smołka [9], Ochal [25] and Migorski [18]. The contact problems for viscoelastic bodies have been recently 
investigated in several papers, see e.g. Chau et al. [3], Jarusek [14], Kuttler and Shillor [16], Rochdi et al. [31], Han and Sofonea [11] and the literature therein. The problem (1) with $A(t, \cdot)$ being multivalued maximal monotone, $B$ coercive and $J \equiv 0$ was considered by Banks et al. [1] in the connection with identification of the damping term in the forced wave equation.

The goal of this paper is to provide the existence and uniqueness results for the problem (1). The main existence result can be proved in two steps (cf. [5]). First we assume regular initial data and reduce this problem to an evolution inclusion of the first order. The latter is solved by using a surjectivity result for multivalued operators of pseudomonotone type. In the second step we remove the restriction on the initial data and we are able to show the result in its generality. The uniqueness of a solution to (1) is obtained in a case when the damping operator is strongly monotone and the subdifferential operator satisfies a relaxed monotonocity condition.

The paper is organized as follows. In Section 1 we present a contact problem of viscoelasticity which serves as a model for the problem (1). In Section 2 we recall some necessary notation and present a result on properties of the Nemitsky operator corresponding to the damping operator. The main results of this paper are delivered in Section 3.

\section{Motivation}

In this section we describe shortly the classical contact model of viscoelasticity and we present its variational form.

We consider a deformable viscoelastic body which occupies a bounded open subset $\Omega \subset \mathbb{R}^{d}, d=2,3$. We suppose that the boundary $\Gamma=\partial \Omega$ is Lipschitz continuous and $\Gamma$ is divided into three mutually disjoint measurable parts $\Gamma_{D}, \Gamma_{N}$ and $\Gamma_{C}$ such that meas $\left(\Gamma_{D}\right)>0$. The body is clamped on $\Gamma_{D}$, so the displacement field vanishes there. Volume forces of density $f_{1}$ act in $\Omega$ and surface tractions of density $f_{2}$ are applied on $\Gamma_{N}$. The body may come in contact with a foundation over the potential contact surface $\Gamma_{C}$. We put $Q=\Omega \times(0, T)$ for $0<T<\infty$. We denote by $u: Q \rightarrow \mathbb{R}^{d}$ the displacement field, by $\sigma: Q \rightarrow \mathcal{S}_{d}$ the stress tensor and by $\varepsilon(u)=\left(\varepsilon_{i j}(u)\right), \varepsilon_{i j}(u)=\frac{1}{2}\left(u_{i, j}+u_{j, i}\right)$ the strain tensor, where $i, j=1, \ldots, d$ and $\mathcal{S}_{d}$ denotes the space $\mathbb{R}_{s}^{d \times d}$ of symmetric matrices of order $d)$.

We suppose the following multivalued counterpart of the Kelvin-Voigt viscoelastic constitutive relation

$$
\sigma\left(u, u^{\prime}\right) \in \mathcal{C}\left(\varepsilon\left(u^{\prime}\right)\right)+\mathcal{G}(\varepsilon(u))
$$


where $\mathcal{C}$ and $\mathcal{G}$ are prescribed multivalued nonlinear and single valued linear constitutive maps, respectively. We remark that in the classical linear viscoelasticity the above law takes the form $\sigma_{i j}=c_{i j k l} \varepsilon_{k l}\left(u^{\prime}\right)+$ $g_{i j k l} \varepsilon_{k l}(u)$, where $\mathcal{C}=\left\{c_{i j k l}\right\}$ and $\mathcal{G}=\left\{g_{i j k l}\right\}, i, j, k, l=1, \ldots, d$ are the viscosity and elasticity tensors, respectively.

We denote by $u_{N}$ and $u_{T}$ the normal and the tangential components of the displacement $u$ on $\Gamma, u_{N}=u \cdot n, u_{T}=u-u_{N} n$, where $n$ is the outward unit vector to $\Gamma$. Similarly, the normal and the tangential components of the stress field on $\Gamma$ are given by $\sigma_{N}=(\sigma n) \cdot n$ and $\sigma_{T}=\sigma n-\sigma_{N} n$, respectively. On the contact surface $\Gamma_{C}$ we consider the following subdifferential boundary conditions. The normal stress $\sigma_{N}$ and the normal displacement $u_{N}$ satisfy the nonmonotone normal compliance response condition of the form

$$
-\sigma_{N} \in \partial j_{N}\left(x, t, u_{N}\right) \text { on } \Gamma_{C} \times(0, T) .
$$

The friction law between the friction force $\sigma_{T}$ and the tangential displacement $u_{T}$ on $\Gamma_{C}$ is given by

$$
-\sigma_{T} \in \partial j_{T}\left(x, t, u_{T}\right) \text { on } \Gamma_{C} \times(0, T) .
$$

Here $j_{N}: \Gamma_{C} \times(0, T) \times \mathbb{R} \rightarrow \mathbb{R}$ and $j_{T}: \Gamma_{C} \times(0, T) \times \mathbb{R}^{d} \rightarrow \mathbb{R}$ are locally Lipschitz functions in their last variables and $\partial j_{N}, \partial j_{T}$ represent the Clarke subdifferentials of $j_{N}(x, t, \cdot)$ and $j_{T}(x, t, \cdot)$, respectively. These boundary conditions include as special cases the classical boundary conditions of (see e.g. Panagiotopoulos [27], Chapter 2.3 and Naniewicz and Panagiotopoulos [24]).

Let us denote by $u_{0}$ and $u_{1}$ the initial displacement and the initial velocity. The classical formulation of the contact problem is stated as follows: find $u: Q \rightarrow \mathbb{R}^{d}$ and $\sigma: Q \rightarrow \mathcal{S}_{d}$ such that

$$
\begin{cases}u^{\prime \prime}-\operatorname{div} \sigma=f_{1} & \text { in } Q \\ \sigma \in \mathcal{C}\left(\varepsilon\left(u^{\prime}\right)\right)+\mathcal{G}(\varepsilon(u)) & \text { in } Q \\ u=0 & \text { on } \Gamma_{D} \times(0, T) \\ \sigma n=f_{2} & \text { on } \Gamma_{N} \times(0, T) \\ -\sigma_{N} \in \partial j_{N}\left(x, t, u_{N}\right),-\sigma_{T} \in \partial j_{T}\left(x, t, u_{T}\right) & \text { on } \Gamma_{C} \times(0, T) \\ u(0)=u_{0}, \quad u^{\prime}(0)=u_{1} & \text { in } \Omega .\end{cases}
$$

In order to give a variational formulation of this problem let $H=$ $L^{2}\left(\Omega ; \mathbb{R}^{d}\right), \mathcal{H}=L^{2}\left(\Omega ; \mathcal{S}_{d}\right), H_{1}=\{u \in H: \varepsilon(u) \in \mathcal{H}\}=H^{1}\left(\Omega ; \mathbb{R}^{d}\right)$ and $V=\left\{v \in H_{1}: v=0\right.$ on $\left.\Gamma_{D}\right\}$. Using the Green formula, the definition of the Clarke subdifferential and assuming the suitable regularity of the 
data (cf. Denkowski and Migórski [5] for details), we obtain the following variational formulation of (4): find $u:(0, T) \rightarrow V$ and $\sigma:(0, T) \rightarrow \mathcal{H}$ such that

$$
\left\{\begin{aligned}
\left\langle u^{\prime \prime}(t)\right. & , v\rangle_{V^{*} \times V}+(\sigma(t), \varepsilon(v))_{\mathcal{H}}+ \\
& +\int_{\Gamma_{C}}\left(j_{N}^{0}\left(x, t, u_{N} ; v_{N}\right)+j_{T}^{0}\left(x, t, u_{T} ; v_{T}\right)\right) d \Gamma(x) \geq \\
& \geq\langle f(t), v\rangle_{V^{*} \times V} \quad \text { for all } v \in V \text { and a.e. } t \in(0, T) \\
\sigma(t) & \in \mathcal{C}\left(\varepsilon\left(u^{\prime}(t)\right)\right)+\mathcal{G}(\varepsilon(u(t))) \text { for a.e. } t \in(0, T) \\
u(0) & =u_{0}, u^{\prime}(0)=u_{1},
\end{aligned}\right.
$$

where

$$
\langle f(t), v\rangle_{V^{*} \times V}=\left(f_{1}(t), v\right)_{H}+\left(f_{2}(t), v\right)_{L^{2}\left(\Gamma_{N} ; \mathbb{R}^{d}\right)} \text { for } v \in V \text { and a.e. } t \text {. }
$$

Let $\mathcal{V}=L^{2}(0, T ; V), \mathcal{W}=\left\{w \in \mathcal{V}: w^{\prime} \in \mathcal{V}^{*}\right\}$ and let $\bar{\gamma}: H^{\delta}\left(\Omega ; \mathbb{R}^{d}\right) \rightarrow$ $H^{1 / 2}\left(\Gamma ; \mathbb{R}^{d}\right) \subset L^{2}\left(\Gamma ; \mathbb{R}^{d}\right)$ be the trace operator where $\delta \in(1 / 2,1)$. We define the operators $A:(0, T) \times V \rightarrow 2^{V^{*}}$ and $B: V \rightarrow V^{*}$ by

$$
\begin{gathered}
\langle A(t, u), v\rangle_{V^{*} \times V}=(\mathcal{C}(x, t, \varepsilon(u)), \varepsilon(v))_{\mathcal{H}} \quad \text { for } u, v \in V \text { and } t \in(0, T), \\
\langle B u, v\rangle_{V^{*} \times V}=(\mathcal{G}(x, t, \varepsilon(u)), \varepsilon(v))_{\mathcal{H}} \quad \text { for } u, v \in V \text { and } t \in(0, T)
\end{gathered}
$$

and the functional $J:(0, T) \times L^{2}\left(\Gamma_{C} ; \mathbb{R}^{d}\right) \rightarrow \mathbb{R}$ by

$$
J(t, v)=\int_{\Gamma_{C}}\left(j_{N}\left(x, t, v_{N}(x)\right)+j_{T}\left(x, t, v_{T}(x)\right)\right) d \Gamma(x)
$$

for $t \in(0, T)$ and $v \in L^{2}\left(\Gamma_{C} ; \mathbb{R}^{d}\right)$. Consider now the following inclusion

$$
\left\{\begin{array}{l}
\text { find } u \in \mathcal{V} \text { with } u^{\prime} \in \mathcal{W} \text { such that } \\
u^{\prime \prime}(t)+A\left(t, u^{\prime}(t)\right)+B u(t)+\bar{\gamma}^{*}(\partial J(t, \bar{\gamma} u(t))) \ni f(t) \text { a.e. } t \\
u(0)=u_{0}, u^{\prime}(0)=u_{1}
\end{array}\right.
$$

where $\bar{\gamma}^{*}$ denotes the adjoint operator to $\bar{\gamma}$. It can be shown (cf. Denkowski and Migórski [5] for details) that every solution to the inclusion (6) is also a solution to the problem (5). Therefore in what follows we are interested in the existence result for a problem of type (6).

\section{Preliminaries}

In this section we recall some definitions needed in the sequel and state a result that shows that certain properties of the damping mapping can be lifted to its Nemitsky operator. 
Let $V$ be a reflexive separable Banach space. We denote by $\langle\cdot, \cdot\rangle$ the pairing between $V$ and its dual $V^{*}$.

Definition 1 A multivalued operator $T: V \rightarrow 2^{V^{*}}$ is said to be pseudomonotone if the following conditions hold:

(j) the set $T v$ is nonempty, bounded, closed and convex for all $v \in V$;

(jj) $T$ is usc from each finite dimensional subspace of $V$ into $V^{*}$ endowed with the weak topology;

(jjj) if $v_{n} \in V, v_{n} \rightarrow v$ weakly in $V$ and $v_{n}^{*} \in T v_{n}$ is such that $\limsup \left\langle v_{n}^{*}, v_{n}-v\right\rangle \leq 0$, then to each $y \in V$, there exists $v^{*}(y) \in$ Tv such that $\left\langle v^{*}(y), v-y\right\rangle \leq \liminf \left\langle v_{n}^{*}, v_{n}-y\right\rangle$.

Lemma 2 Assume that a multivalued operator $T: V \rightarrow 2^{V^{*}}$ satisfies conditions $(j)$ and $(j j)$ of the definition of pseudomonotonicity and $T$ is bounded (i.e. it maps bounded sets into bounded sets). Then $T$ is usc with respect to the strong topology in $V$ and the weak topology in $V^{*}$.

For the proof we refer to Lemma 1.4 in Kuttler [15].

We now comment on a measurability condition for multivalued mappings. For the following definitions, see Section 1.0 of Hu and Papageorgiou [13].

Definition $3 A$ multifunction $F:(0, T) \rightarrow 2^{V^{*}}$ is said to be measurable, if for every $U \subset V^{*}$ open, we have $F^{-}(U)=\{t \in(0, T)$ : $F(t) \cap U \neq \emptyset\}$ is measurable.

Definition 4 A multifunction $S:(0, T) \times V \rightarrow 2^{V^{*}}$ is said to be (strongly) measurable, if for every $C \subset V^{*}$ closed, we have $\{(t, v) \in(0, T) \times V$ : $S(t, v) \cap C \neq \emptyset\}$ is a Borel set in $(0, T) \times V$.

The following result due to Kuttler [15], Lemma 5.3 shows that the strong measurability condition implies a kind of measurability condition for multivalued operators which is useful in our setting.

Lemma 5 Suppose $S:(0, T) \times V \rightarrow 2^{V^{*}}$ has nonempty, closed, convex values and it satisfies the measurability condition of Definition 4 for every $C \subset V^{*}$ closed convex set. Then

$\left(^{*}\right)$ for every $\alpha:(0, T) \rightarrow \mathbb{R}$ measurable and $x, y:(0, T) \rightarrow V$ measurable, the multifunction $F:(0, T) \rightarrow 2^{V^{*}}$ defined by

$$
F(t)=\{w \in S(t, x(t)):\langle w, x(t)-y(t)\rangle \leq \alpha(t)\}
$$


is measurable (in the sense of Definition 3).

In what follows (see condition $H(A)(i i)$ below) instead of the standard definition (Definition 4) of measurability of a multivalued operator, we assume it satisfies condition $(*)$ of Lemma 5 , which will be sufficient for our purposes.

Given $2 \leq p<\infty$ we introduce the spaces $\mathcal{V}=L^{p}(0, T ; V), \mathcal{V}^{*}=$ $L^{q}\left(0, T ; V^{*}\right), 1 / p+1 / q=1$ and we denote by $\langle\langle\cdot, \cdot\rangle\rangle$ the duality between $\mathcal{V}$ and $\mathcal{V}^{*}$

Definition 6 Let $A:(0, T) \times V \rightarrow 2^{V^{*}}$ be a multivalued operator. The operator $\mathcal{A}: \mathcal{V} \rightarrow 2^{\mathcal{V}^{*}}$ given by $\mathcal{A} v=\left\{z \in \mathcal{V}^{*}: z(t) \in A(t, v(t))\right.$ a.e. $t \in(0, T)\}$ for $v \in \mathcal{V}$ is called the Nemitsky operator corresponding to A.

We recall also the notion of $L$-pseudomonotonicity (see e.g. [6]). Let $L: D(L) \subset \mathcal{V} \rightarrow \mathcal{V}^{*}$ be a linear maximal monotone operator.

Definition 7 We say that the operator $\mathcal{A}: \mathcal{V} \rightarrow 2^{\mathcal{V}^{*}}$ is L-pseudomonotone, if the following conditions hold:

(k) the set $\mathcal{A} v$ is nonempty, weakly compact and convex for all $v \in \mathcal{V}$;

(kk) $\mathcal{A}$ is usc from each finite dimensional subspace of $\mathcal{V}$ into $\mathcal{V}^{*}$ furnished with the weak topology;

$(k k k)$ if $\left\{v_{n}\right\} \subset D(L), v_{n} \rightarrow v$ weakly in $\mathcal{V}, L v_{n} \rightarrow L v$ weakly in $\mathcal{V}^{*}$, $v_{n}^{*} \in \mathcal{A} v_{n}, v_{n}^{*} \rightarrow v^{*}$ weakly in $\mathcal{V}^{*}$ and $\lim \sup \left\langle\left\langle v_{n}^{*}, v_{n}-v\right\rangle\right\rangle \leq 0$, then $v^{*} \in \mathcal{A} v$ and $\left\langle\left\langle v_{n}^{*}, v_{n}\right\rangle\right\rangle \rightarrow\left\langle\left\langle v^{*}, v\right\rangle\right\rangle$.

The following result generalizes Theorem 2(b) of Berkovits and Mustonen [2].

THEOREM 8 Assume that a multivalued operator satisfies the following hypothesis:

$\underline{H(A)}: \quad A:(0, T) \times V \rightarrow 2^{V^{*}}$ is a multivalued operator such that

(i) $A(t, \cdot)$ satisfies conditions ( $j)$ and (jjj) in the definition of pseudomonotone operator;

(ii) $A$ is measurable in the sense of condition (*);

(iii) there are $a_{1} \in L^{q}(0, T)$ and $b_{1}>0$ such that $\left\|v^{*}\right\|_{V^{*}} \leq a_{1}(t)+$ $b_{1}\|v\|_{V}^{p-1}$ for all $v^{*} \in A(t, v), v \in V$ and a.e. $t \in(0, T)$;

(iv) there are constants $\beta_{1}>0, \beta_{2} \geq 0, r \in(0, p)$ and a function $a \in L^{1}(0, T)$ such that $\left\langle v^{*}, v\right\rangle \geq \beta_{1}\|v\|_{V}^{p}-\beta_{2}\|v\|_{V}^{r}-a(t)$ for all $v^{*} \in A(t, v), v \in V$ and a.e. $t \in(0, T)$. 
Then the Nemitsky operator $\mathcal{A}$ corresponding to $A$ has the following properties:

(1) there are constants $\bar{a}_{1} \geq 0$ and $\bar{b}_{1}>0$ such that $\left\|v^{*}\right\| \mathcal{V}^{*} \leq \bar{a}_{1}+$ $\bar{b}_{1}\|v\|_{\mathcal{V}}^{p-1}$ for all $v^{*} \in \mathcal{A} v$ and $v \in \mathcal{V}$;

(2) there are constants $\bar{\beta}_{2} \geq 0$ and $\bar{a}>0$ such that $\left\langle\left\langle v^{*}, v\right\rangle\right\rangle \geq \beta_{1}\|v\|_{\mathcal{V}}^{p}-$ $\bar{\beta}_{2}\|v\|_{\mathcal{V}}-\bar{a}$ for all $v^{*} \in \mathcal{A} v$ and $v \in \mathcal{V}$;

(3) $\mathcal{A}$ is L-pseudomonotone, where $L: D(L) \subset \mathcal{V} \rightarrow \mathcal{V}^{*}$ is given by $L v=v^{\prime}$ for all $v \in D(L)=\left\{v \in \mathcal{V}: v^{\prime} \in \mathcal{V}^{*}, v(0)=0\right\}$.

The detailed proof of this theorem can be found in Denkowski and Migórski [5]. We conclude this section by recalling (cf. Clarke [4]) the definitions of the generalized directional derivative and the generalized gradient of Clarke for a locally Lipschitz function $h: E \rightarrow \mathbb{R}$, where $E$ is a Banach space. The generalized directional derivative of $h$ at $x \in E$ in the direction $v \in E$, denoted by $h^{0}(x ; v)$, is defined by

$$
h^{0}(x ; v)=\limsup _{y \rightarrow x, t \downarrow 0} \frac{h(y+t v)-h(y)}{t} .
$$

The generalized gradient of $h$ at $x$, denoted by $\partial h(x)$, is a subset of a dual space $E^{*}$ given by $\partial h(x)=\left\{\zeta \in E^{*}: h^{0}(x ; v) \geq\langle\zeta, v\rangle_{E^{*} \times E}\right.$ for all $v \in E\}$.

\section{Existence Theorem}

In this section we deliver the main result of the paper on the existence of solutions to dynamic hemivariational inequalities.

Let $V$ and $Z$ be two reflexive, separable Banach spaces and let $H$ be a Hilbert space. Suppose that $V \subset Z \subset H \approx H^{*} \subset Z^{*} \subset V^{*}$, where $H^{*}$, $Z^{*}$ and $V^{*}$ denote dual spaces to $H, Z$ and $V$, respectively. We assume that all embeddings are dense and continuous, and $V \subset Z$ compactly. We denote by $\langle\cdot, \cdot\rangle$ the duality of $V$ and $V^{*}$ and the pairing between $Z$ and $Z^{*}$ as well. We introduce the following spaces:

$$
\begin{gathered}
\mathcal{V}=L^{p}(0, T ; V), \quad \mathcal{Z}=L^{p}(0, T ; Z), \quad \mathcal{H}=L^{2}(0, T ; H), \\
\mathcal{Z}^{*}=L^{q}\left(0, T ; Z^{*}\right), \quad \mathcal{V}^{*}=L^{q}\left(0, T ; V^{*}\right) \quad \text { with } 1 / p+1 / q=1
\end{gathered}
$$

with some $2 \leq p<\infty$ and $\mathcal{W}=\left\{v \in \mathcal{V}: v^{\prime} \in \mathcal{V}^{*}\right\}$, where the time derivative involved in the definition of $\mathcal{W}$ is understood in the sense of vector valued distributions. We have $\mathcal{W} \subset \mathcal{V} \subset \mathcal{Z} \subset \mathcal{H} \subset \mathcal{Z}^{*} \subset \mathcal{V}^{*}$ with dense and continuous embeddings. Since we have assumed $V \subset Z$ 
compactly, we also know (cf. Theorem 5.1, p.58, Lions [17]) that $\mathcal{W} \subset \mathcal{Z}$ compactly. Moreover, $\mathcal{W} \subset C(0, T ; H)$ is continuous. The pairing of $\mathcal{V}$ and $\mathcal{V}^{*}$ and also the duality between $\mathcal{Z}$ and $\mathcal{Z}^{*}$ are denoted by $\langle\langle f, g\rangle\rangle=$ $\int_{0}^{T}\langle f(t), g(t)\rangle d t$

Consider the following initial value problem for evolution hemivariatonal inequality of second order:

$$
\left\{\begin{array}{l}
\text { find } y \in \mathcal{V} \text { such that } y^{\prime} \in \mathcal{W} \text { and } \\
y^{\prime \prime}(t)+A\left(t, y^{\prime}(t)\right)+B y(t)+\partial J(t, y(t)) \ni f(t) \text { a.e. } t \in(0, T) \\
y(0)=y_{0}, y^{\prime}(0)=y_{1}
\end{array}\right.
$$

The problem $(7)$ is called hemivariational inequality since it is equivalent to the following one:

$$
\left\{\begin{array}{l}
\text { find } y \in \mathcal{V} \text { with } y^{\prime} \in \mathcal{W} \text { such that there is } \eta \in \mathcal{V}^{*} \text { satisfying } \\
\left\langle y^{\prime \prime}(t)+\eta(t)+B y(t)-f(t), v\right\rangle+J^{0}(t, y(t) ; v) \geq 0 \\
\quad \text { for all } v \in V \text { and a.e. } t \in(0, T) \\
\eta(t) \in A\left(t, y^{\prime}(t)\right) \text { a.e. } t \in(0, T) \\
y(0)=y_{0}, y^{\prime}(0)=y_{1},
\end{array}\right.
$$

where $J^{0}(t, v ; w)$ is the generalized directional derivative of $J(t, \cdot)$ at a point $v \in Z$ in the direction $w \in Z$.

Definition 9 An element $y \in \mathcal{V}$ solves (7) if and only if $y^{\prime} \in \mathcal{W}$ and there exist $\eta \in \mathcal{V}^{*}$ and $\zeta \in \mathcal{Z}^{*}$ such that

$$
\left\{\begin{array}{l}
y^{\prime \prime}(t)+\eta(t)+B y(t)+\zeta(t)=f(t) \text { a.e. } t \in(0, T) \\
\eta(t) \in A\left(t, y^{\prime}(t)\right), \quad \zeta(t) \in \partial J(t, y(t)) \quad \text { a.e. } t \in(0, T) \\
y(0)=y_{0}, y^{\prime}(0)=y_{1} .
\end{array}\right.
$$

We admit the following hypotheses:

$\underline{H(B)}: \quad B: V \rightarrow V^{*}$ is a bounded, linear, positive and symmetric operator;

$\underline{H(J)}: \quad J:(0, T) \times Z \rightarrow \mathbb{R}$ is a function such that

(i) for each $z \in Z$, the map $J(\cdot, z)$ is measurable and $J(\cdot, 0) \in L^{1}(0, T)$;

(ii) for each $t \in(0, T)$, the function $J(t, \cdot)$ is locally Lipschitz;

(iii) there exists $\bar{c}>0$ such that for all $\zeta \in \partial J(t, z), z \in Z$ and $t \in(0, T)$, we have $\|\zeta\|_{Z^{*}} \leq \bar{c}\left(1+\|z\|_{Z}^{2 / q}\right) ;$ 
$\underline{\left(H_{0}\right)}: \quad f \in \mathcal{V}^{*}, y_{0} \in V, y_{1} \in H$

$\underline{\left(H_{1}\right)}:$ If $p=2$, then $\beta_{1}>\bar{c} \beta^{2} T$, where $\beta>0$ is an embedding constant of $V$ into $Z$.

We start the study of (7) with the a priori estimates for the solutions.

Lemma 10 Assume that $H(A), H(B), H(J)$ and $\left(H_{0}\right)$ hold and $y$ is a solution to (7). If $p>2$, then there is a constant $C>0$ such that

$$
\|y\|_{C(0, T ; V)}+\left\|y^{\prime}\right\|_{\mathcal{W}} \leq C\left(1+\left\|y_{0}\right\|_{V}^{2 / q}+\left\|y_{1}\right\|_{H}^{2 / q}+\|f\|_{\mathcal{V}^{*}}^{2 / q}\right) .
$$

Moreover, the estimate (8) still holds for $p=2$ provided $\left(H_{1}\right)$ is satisfied.

If $Z=H$, then the estimate (8) holds for $p \geq 2$ without the hypothesis $\left(H_{1}\right)$. Namely, we have

Lemma 11 If $H(A), H(B), H(J)$ and $\left(H_{0}\right)$ hold, $p \geq 2$ and $Z=H$, then for every $y$ solution to (7), the estimate (8) holds.

The main result of this paper is the following

ThEOREM 12 If hypotheses $H(A), H(B), H(J),\left(H_{0}\right)$ and $\left(H_{1}\right)$ hold, then the problem (7) has at least one solution.

The idea of the proof is as follows. First, we consider the operator $K: \mathcal{V} \rightarrow C(0, T ; V)$ given by $K v(t)=\int_{0}^{t} v(s) d s+y_{0}$. Using $K$ we rewrite (7) in the form: find $z \in \mathcal{W}$ such that

$$
\left\{\begin{array}{l}
z^{\prime}(t)+A(t, z(t))+B(K z(t))+\partial J(t, K z(t)) \ni f(t) \text { a.e. } t \\
z(0)=y_{1} .
\end{array}\right.
$$

We observe that $z \in \mathcal{W}$ is a solution to (9) iff $y=K z$ solves (7). We deal now with the problem (9) under the additional hypothesis $y_{1} \in V$. We define the following operators $\mathcal{A}_{1}: \mathcal{V} \rightarrow 2^{\mathcal{V}^{*}}, \mathcal{B}_{1}: \mathcal{V} \rightarrow \mathcal{V}^{*}$ and $\mathcal{N}_{1}: \mathcal{V} \rightarrow$ $2^{\nu^{*}}$ by

$$
\begin{gathered}
\mathcal{A}_{1} v=\left\{v^{*} \in \mathcal{V}^{*}: v^{*}(t) \in A\left(t, v(t)+y_{1}\right) \text { a.e. } \mathrm{t}\right\} \\
\left(\mathcal{B}_{1} v\right)(t)=B\left(K\left(v(t)+y_{1}\right)\right)
\end{gathered}
$$

and

$$
\mathcal{N}_{1} v=\left\{z \in \mathcal{Z}^{*}: z(t) \in \partial J\left(t, K\left(v(t)+y_{1}\right)\right) \text { a.e. } \mathrm{t}\right\}
$$

for all $v \in \mathcal{V}$, respectively. Using these operators, from (9) we have

$$
\left\{\begin{array}{l}
z^{\prime}+\mathcal{A}_{1} z+\mathcal{B}_{1} z+\mathcal{N}_{1} z \ni f \quad \text { a.e. } t \in(0, T) \\
z(0)=0
\end{array}\right.
$$


We note that $z \in \mathcal{W}$ solves (9) iff $z-y_{1} \in \mathcal{W}$ solves (10). Next, defining $L: D(L) \subset \mathcal{V} \rightarrow \mathcal{V}^{*}$ and $\mathcal{T}: \mathcal{V} \rightarrow 2^{\mathcal{V}^{*}}$ by $L z=z^{\prime}$ with $D(L)=\{z \in \mathcal{W}:$ $z(0)=0\}$ and $\mathcal{T} z=\left(\mathcal{A}_{1}+\mathcal{B}_{1}+\mathcal{N}_{1}\right) z$, respectively, the problem (10) takes the form: find $z \in D(L)$ such that $(L+\mathcal{T}) z \ni f$. In order to show the existence of solutions, we can prove that $\mathcal{T}$ is bounded, coercive and $L$-pseudomonotone, and apply a surjectivity result (cf. Theorem 1.3.73 in [6]). Finally, we suppose $y_{1} \in H$ and we establish the existence of solutions in this case.

The existence of solution to (6) can be obtained analogously as for the model problem (7). This follows from the fact that the map $R:(0, T) \times$ $Z \rightarrow 2^{Z^{*}}$ given by $R(t, z)=\bar{\gamma}^{*}(\partial J(t, \bar{\gamma} z(t)))$ has the same properties as $\partial J(t, z)$ (convex and weak compactness of the values, the strong-weak closedness of the graph and a growth condition).

\section{References}

[1] H.T. Banks, S. Reich, and I.G. Rosen. Estimation of nonlinear damping in second order distributed parameter systems. Contral - Theory and Advanced Techn., 6:395-415, 1990.

[2] J. Berkovits and V. Mustonen. Monotonicity methods for nonlinear evolution equations. Nonlinear Analysis, 27:1397-1405, 1996.

[3] O Chau, W. Han, and M. Sofonea. A dynamic frictional contact problem with normal damped response. Acta Appl. Math., 71:159-178, 2002.

[4] F.H. Clarke. Optimization and Nonsmooth Analysis. Wiley - Interscience, New York, 1983.

[5] Z. Denkowski and S. Migórski. Existence of solutions to evolution second order hemivariational inequalities with multivalued damping, submitted.

[6] Z. Denkowski, S. Migórski, and N.S. Papageorgiou. An Introduction to Nonlinear Analysis: Applications. Kluwer Academic/Plenum Publishers, Boston, Dordrecht, London, New York, 2003.

[7] G. Duvaut and J.L. Lions. Les Inéquations en Mécanique et en Physique. Dunod, Paris, 1972.

[8] L. Gasiński. Hyperbolic Hemivariational Inequalities and their Applications to Optimal Shape Design. PhD thesis, Jagiellonian University, Cracow, Poland, 2000.

[9] L. Gasiński and M. Smołka. An existence theorem for wave-type hyperbolic hemivariational inequalities. Math. Nachr., 242:1-12, 2002.

[10] D. Goeleven, M. Miettinen, and P.D. Panagiotopoulos. Dynamic hemivariational inequalities and their applications. J. Optimiz. Theory and Appl., 103:567-601, 1999.

[11] W. Han and M. Sofonea. Quasistatic Contact Problems in Viscoelasticity and Viscoplasticity. AMS and International Press, 2002. 
[12] J. Haslinger, M. Miettinen, and P.D. Panagiotopoulos. Finite Element Method for Hemivariational Inequalities. Theory, Methods and Applications. Kluwer Academic Publishers, Boston, Dordrecht, London, 1999.

[13] S. Hu and N.S. Papageorgiou. Handbook of Multivalued Analysis, Volume I: Theory. Kluwer, Dordrecht, 1997.

[14] J. Jarusek. Dynamic contact problems with given friction for viscoelastic bodies. Czech. Math. J., 46:475-487, 1996.

[15] K. Kuttler. Non-degenerate implicit evolution inclusions. Electronic J. Diff. Equations, 34:1-20, 2000.

[16] K.L. Kuttler and M. Shillor. Set-valued pseudomonotone maps and degenerate evolution inclusions. Comm. Contemp. Math., 1:87-123, 1999.

[17] J.L. Lions. Quelques méthodes de résolution des problémes aux limites non linéaires. Dunod, Paris, 1969.

[18] S. Migórski. Boundary hemivariational inequalities of hyperbolic type and applications, J. Global Optim., in press, 2004.

[19] S. Migórski. Dynamic hemivariational inequality modeling viscoelastic contact problem with normal damped response and friction. Applicable Analysis, in press, 2004.

[20] S. Migórski. Existence and convergence results for evolution hemivariational inequalities. Topological Methods Nonlinear Anal., 16:125-144, 2000.

[21] S. Migórski. Evolution hemivariational inequalities in infinite dimension and their control. Nonlinear Analysis, 47:101-112, 2001.

[22] S. Migórski. Modeling, Analysis and Optimal Control of Systems Governed by Hemivariational Inequalities, pp. 248-279, chapter in the book "Industrial Mathematics and Statistics" dedicated to commemorate the Golden Jubilee of Indian Institute of Technology, Kharagpur, India, 2002, J.C. Misra, ed., Narosa Publishing House. Delhi, 2003.

[23] D. Motreanu and P.D. Panagiotopoulos. Minimax Theorems and Qualitative Properties of the Solutions of Hemivariational Inequalities and Applications. Kluwer Academic Publishers, Boston, Dordrecht, London, 1999.

[24] Z. Naniewicz and P.D. Panagiotopoulos. Mathematical Theory of Hemivariational Inequalities and Applications. Marcel Dekker, Inc., New York, Basel, Hong Kong, 1995.

[25] A. Ochal. Optimal Control of Evolution Hemivariational Inequalities. PhD thesis, Jagiellonian University, Cracow, Poland, 2001.

[26] P.D. Panagiotopoulos. Inequality Problems in Mechanics and Applications. Convex and Nonconvex Energy Functions. Birkhäuser, Basel, 1985.

[27] P.D. Panagiotopoulos. Hemivariational Inequalities, Applications in Mechanics and Engineering. Springer-Verlag, Berlin, 1993.

[28] P.D. Panagiotopoulos. Hemivariational inequalities and fan-variational inequalities. new applications and results. Atti Sem. Mat. Fis. Univ. Modena, 43:159191, 1995.

[29] P.D. Panagiotopoulos. Modelling of nonconvex nonsmooth energy problems: dynamic hemivariational inequalities with impact effects. J. Comput. Appl. Math., 63:123-138, 1995. 
[30] P.D. Panagiotopoulos and G. Pop. On a type of hyperbolic variationalhemivariational inequalities. J. Applied Anal., 5 (1):95-112, 1999.

[31] M. Rochdi, M. Shillor, and M. Sofonea. A quasistatic contact problem with directional friction and damped response. Applicable Analysis, 68:409-422, 1998. 